\title{
The metabolic syndrome: the crossroads of diet and genetics
}

\author{
Helen M. Roche*, Catherine Phillips and Michael J. Gibney \\ Nutrigenomics Research Group, Department of Clinical Medicine, Institute of Molecular Medicine, \\ Trinity College Dublin, Dublin 8, Republic of Ireland
}

\begin{abstract}
The metabolic syndrome is a very common disease associated with an increased risk of type 2 diabetes mellitus (T2DM) and CVD. The clinical characteristics of the metabolic syndrome include insulin resistance, dyslipidaemia, abdominal obesity and hypertension. The diverse clinical characteristics illustrate the complexity of the disease process, which involves several dysregulated metabolic pathways. Thus, multiple genetic targets must be involved in the pathogenesis and progression of the metabolic syndrome. Nevertheless, the human genome has not changed markedly in the last decade but the prevalence of the metabolic syndrome has increased exponentially, which illustrates the importance of gene-environmental interactions. There is good evidence that nutrition plays an important role in the development and progression of the metabolic syndrome. Indeed, obesity is a key aetiological factor in the development of the metabolic syndrome. Understanding the biological impact of gene-nutrient interactions will provide a key insight into the pathogenesis and progression of diet-related polygenic disorders, including the metabolic syndrome. The present paper will explore the interactions between genetic background and dietary exposure or nutritional therapy, focusing on the role of dietary fatty acids within the context of nutrient regulation of gene expression and individual responsiveness to dietary therapy. Only with a full understanding of gene-gene, gene-nutrient and gene-nutrient-environment interactions can the molecular basis of the metabolic syndrome be solved to minimise the adverse health effects of obesity and reduce the risk of the metabolic syndrome, and subsequent T2DM and CVD.
\end{abstract}

Metabolic syndrome: Obesity: Dietary fatty acids: Nutrient regulation of gene expression: Insulin resistance

The metabolic syndrome represents a heterogeneous group of metabolic disorders, the central component of which is insulin resistance. It can involve multiple metabolic pathways that culminate in dyslipidaemia, abdominal obesity, microalbuminuria and hypertension. The WHO (Alberti \& Zimmet, 1998) has defined the metabolic syndrome as impaired insulin sensitivity, glucose intolerance or diabetes mellitus in combination with at least two other metabolic derangements, including abdominal obesity, dyslipidaemia (HDL-cholesterol levels and/or elevated plasma triacylglycerol (TAG) concentrations) and urinary microalbuminuria. The National Cholesterol Education Program Adult Treatment Panel III (Expert Panel on Detection, Evaluation, and Treatment of High Blood Cholesterol in Adults, 2001) have proposed that individuals with the metabolic syndrome have three or more of the following metabolic derangements, including central obesity (waist girth $>1020 \mathrm{~mm}$ in men or $>880 \mathrm{~mm}$ in women), low HDL-cholesterol concentrations $(<1 \cdot 1 \mathrm{mmol} / \mathrm{l}$ for men or $<1.3 \mathrm{mmol} / \mathrm{l}$ for women), hypertriacylglycerolaemia $(>1.7 \mathrm{mmol} / \mathrm{l})$, hypertension (blood pressure $>130$ / $85 \mathrm{mmHg}$ ) or fasting hyperglycaemia $(>6 \cdot 1 \mathrm{mmol} / \mathrm{l})$. Thus, the metabolic syndrome represents a genetically-heterogeneous group of metabolic disorders sharing insulin resistance. The multiple genetic targets involved in the pathogenesis and progression of the metabolic syndrome could relate to pancreatic $\beta$-cell function, insulin action, glucose metabolism, hypertension, lipid and lipoprotein metabolism etc. (Groop, 2000; Barroso et al. 2003).

In parallel with the increasing incidence of type 2 diabetes mellitus (T2DM), the incidence of obesity and the metabolic syndrome have been rising at an alarming rate worldwide, resulting in increased CVD and premature death (Isomaa et al. 2001). Ford et al. (2004) have reported

\footnotetext{
Abbreviations: CAPN10, Calpain 10; LC, long-chain; P:S, PUFA: saturated fatty acids; SFA, saturated fatty acids; TAG, triacylglycerol; T2DM, type 2 diabetes mellitus.

*Corresponding author: Dr Helen M. Roche, fax +35314542043, email hmroche@tcd.ie
} 


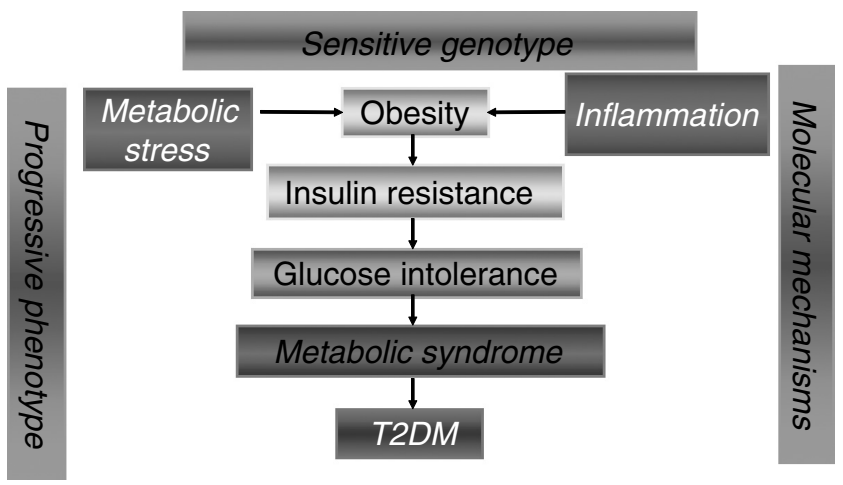

Fig. 1. The development and progression of the metabolic syndrome. T2DM, type 2 diabetes mellitus.

an increase in the prevalence of the metabolic syndrome from $23.1 \%$ in the National Health and Nutrition Examination Survey III (1988-1994) to $26.7 \%$ in the National Health and Nutrition Examination Survey (1999-2000). It is important to note that the human genome has not changed markedly in the last 10 years but the prevalence of the metabolic syndrome has increased exponentially, which illustrates the importance of gene-environmental interactions. Fig. 1 illustrates the concept that the metabolic syndrome is a progressive phenotype, whereby obesity and insulin resistance results in the metabolic syndrome and T2DM in individuals who carry the elusive sensitive genotype. Multiple organs are involved; skeletal muscle, liver and adipose tissue become insulin resistant and fail to promote glucose uptake effectively (Le Marchand-Brustel et al. 2003). Pancreatic $\beta$-cells initially compensate, increasing insulin secretion to maintain euglycaemia, but at some point $\beta$-cells fail to respond effectively, leading to glucose intolerance. Impaired insulin action and hyperglycaemia adversely affect peripheral organ function and promote the development of other metabolic abnormalities characteristic of the metabolic syndrome, including elevated NEFA and TAG concentrations, low HDL levels, hyperglycaemia, increased vascular resistance and a prothrombotic state (Saltiel, 2000). The incidence of the metabolic syndrome is age-dependent; an increase from $6.7 \%$ among participants aged 20-29 years to $43.5 \%$ for participants aged 60-69 years has been reported (Ford et al. 2002). The progressive and age-dependent nature of the disease makes it much harder to define the genetic components of the metabolic syndrome. Nevertheless, with a greater understanding of the molecular mechanisms involved it will be possible to determine how nutrient-derived metabolic (high-fat diet, elevated plasma non-NEFA levels etc.) and pro-inflammatory stressors, in the presence of obesity, induce insulin resistance and result in the metabolic syndrome.

\section{Genetic and environmental components of the metabolic syndrome}

The global increase in the incidence of the metabolic syndrome and T2DM over the last few decades is a salient example of how the interaction between lifestyle and genotype can dramatically impact on health. The familial nature (Rich, 1990; Freeman et al. 2002; Mills et al. 2004), the marked difference in the prevalence among various racial groups (Al-Shaer \& Abusabha, 2005) and the difference in concordance rates between monozygotic twins (Poulsen et al. 2001) is clearly consistent with a genetic component to disease susceptibility. In European populations familial studies show a sibling relative risk of 3.5-4 (Rich, 1990), indicating that siblings of family members with T2DM are up to four times more likely to develop T2DM than the general population. Heritability studies demonstrate a marked genetic influence on several features of the insulin resistance syndrome. One study (Mills et al. 2004) has shown that the heritability estimates for fasting glucose concentration and the homeostasis model assessment of pancreatic $\beta$-cell function are 0.72 and 0.78 respectively. On the other hand, another study (Freeman et al. 2002) has reported heritability estimates for fasting glucose, insulin, TAG and HDLcholesterol concentrations of 20, 23, 20 and $43 \%$ respectively.

Shared genetic and environmental components almost certainly contribute to the predisposition to disease; nevertheless, determining the relative contribution of each factor is difficult and varies for the different components of the metabolic syndrome. Poulsen et al. (2001) have studied the relative impact of genetic $v$. environmental factors for the development of the components of the metabolic syndrome amongst 303 elderly twin pairs. This study has demonstrated that glucose intolerance, obesity and low HDL-cholesterol concentrations are significantly higher among monozygotic twins than among dizygotic twins, indicating a genetic influence on the development on these phenotypes. In contrast, the heritability estimates for hyperinsulinaemia, hypertension and hypertriacylglycerolaemia are low, indicating a more important environmental influence on these components of the metabolic syndrome. These data indicate a major role for genetic susceptibility, whilst also emphasising the important role of the environment. In particular, trends in diet and physical activity, coupled with genetic susceptibility, must account for the recent and dramatic risk in the incidence of the metabolic syndrome and T2DM, demonstrating the important impact of gene-environment interactions.

\section{Diet-related environmental factors and the risk of the metabolic syndrome}

Obesity has been identified as the key aetiological condition that predisposes to the development of the metabolic syndrome. There are at least two ways whereby adipose tissue may influence glucose homeostasis and act as the key aetiological factor in the development of insulin resistance and the metabolic syndrome (Kahn \& Flier, 2000). First, excessive adipose tissue energy storage results in increased fatty acid flux to other tissues and increased TAG storage in peripheral tissues, which interferes with insulin signalling, inhibits glucose uptake and promotes insulin resistance. Second, adipose tissue is an important 
endocrine organ that secretes several inflammatory factors, collectively known as adipocytokines or adipokines, many of which have a direct effect on insulin sensitivity (Trayhurn \& Wood, 2004). There is no doubt that obesity prevention would reduce negative health effects associated with the metabolic syndrome, in particular T2DM and CVD. Nevertheless, current anti-obesity dietary strategies and public health initiatives have been largely unsuccessful, indicating that other strategies are required. Given the increasing prevalence of obesity, there is a high requirement to explore alternative approaches to reduce the impact of the adverse health effects of obesity and the metabolic syndrome.

Several studies have suggested that there may be a link between altered dietary fatty acid composition and risk of the metabolic syndrome. Aside from the obvious link between a high-fat diet and the development of obesity, there is a growing body of evidence that suggests that dietary fatty acid composition could affect insulinmediated insulin action. The relationship between dietary fat composition and the risk of the metabolic syndrome has been recently reviewed in more detail (Roche, 2005). Briefly, a prospective study (Laaksonen et al. 2002) has demonstrated that the proportion of serum esterified and non-esterified saturated fatty acids (SFA) is increased and that of PUFA is decreased in men who after 4 years develop impaired fasting hyperglycaemia or T2DM. Prospective epidemiological evidence (Harding et al. 2004) suggests that increased dietary PUFA:SFA (P:S) is associated with reduced risk of T2DM, independent of age, gender, family history of diabetes and other lifestyle factors. Also, the Nurses' Health Study (Tanasescu et al. 2004) has shown that higher SFA intake and a low dietary $\mathrm{P}: \mathrm{S}$ are related to increased CVD risk among women with T2DM. The latter study has also demonstrated that the replacement of $5 \%$ of the energy from SFA with MUFA is associated with a $37 \%$ lower risk of CVD. A number of dietary intervention studies have investigated the causal nature of the relationship between dietary fatty acid composition and insulin resistance (see Roche, 2005). Most of the earlier dietary intervention studies have shown no association, but these negative results probably reflect poor study design, short study duration, inaccurate measures of insulin sensitivity and/or poor statistical power. More recently, the KANWU study (Vessby et al. 2001) has demonstrated that decreasing dietary SFA and increasing MUFA improves insulin sensitivity but has no effect on insulin secretion. It is interesting to note that within each dietary group a second assignment to fish oil supplementation or placebo was completed, but the addition of long-chain (LC) $n$-3 PUFA was found to have no effect on insulin sensitivity. Furthermore, PerezJimenez et al. (2001) have shown that isoenergetic substitution of SFA by MUFA markedly improves insulin sensitivity. In contrast, Lovejoy et al. (2002) have failed to show any marked effect of high-MUFA, high-SFA or trans-fatty acid-rich diets on insulin sensitivity. However, when the groups were subdivided according to BMI insulin sensitivity was found to be $24 \%$ lower in overweight individuals (BMI $25-30 \mathrm{~kg} / \mathrm{m}^{2}$ ) after the SFA diet, compared with after the MUFA diet. Also, these diets were low in fat $(28 \%$ energy); therefore, the effects of dietary fat composition may not be relevant when accompanied by a background low-fat diet.

A number of positive health benefits relevant to the metabolic syndrome have been associated with increased LC $n-3$ PUFA intake, particularly in relation to TAG metabolism (Roche \& Gibney, 2000). Animal studies show that feeding LC $n-3$ PUFA has positive effects on glucose metabolism and insulin resistance (Storlien et al. 1987; Aguilera et al. 2004). However, in human subjects there are no definitive data that consistently show positive effects of LC $n-3$ PUFA on insulin resistance. Human epidemiological data suggest that habitual dietary fish intake is inversely associated with the incidence of impaired glucose tolerance and T2DM (Feskens et al. 1985, 1991). While some intervention studies have reported positive effects of LC $n-3$ PUFA supplementation on insulin sensitivity in individuals with impaired glucose tolerance and diabetes (Popp-Snijders et al. 1987; Fasching et al. 1991), other studies have not (Vessby et al. 2001; Brady et al. 2004). Clearly, the health impact of increased LC $n$-3 PUFA consumption on insulin resistance in human subjects requires further clarification.

\section{Approaches for identifying the genetic determinants of the metabolic syndrome}

The candidate-gene approach has been the traditional approach for identifying the genes involved in the metabolic syndrome. The candidate genes can be identified according to biological function and/or linkage studies, and association tests for significant differences in their allele frequencies between a patient group and a control population. Given the number of metabolic pathways involved in the metabolic syndrome it is immediately apparent that the number of potential candidate genes is tremendous. Overall, there has not been a high extent of success from candidate-gene studies in terms of defining the genetic determinants of the metabolic syndrome. Genetic-disease-association studies are fraught with difficulties, and a number of the positive results have not been replicated in subsequent studies (Hirschhorn et al. 2002). The main reasons for the disparity are inadequate statistical power, multiple hypothesis testing, population stratification, publication bias and phenotypic differences. It is becoming increasingly evident that the identification of true genetic associations in common multi-factorial conditions, such as the metabolic syndrome, requires large studies consisting of thousands rather than hundreds of subjects. Also, the absence of large single-gene effects and the detection of multiple small effects accentuate the need for the study of larger populations in order to reliably identify the size of the effect now expected for complex diseases.

Recent studies (Barak et al. 1999; Barroso et al. 1999) have suggested guidelines for designing association studies, and implementation of these guidelines has identified apparently promising candidates in T2DM. These studies have focused on variants of the PPAR $\gamma$ gene, which is a strong candidate for conferring susceptibility to the 
metabolic syndrome, because PPAR $\gamma$ encodes a transcription factor that regulates adipogenesis, and lipid and glucose metabolism. In the initial study (Deeb et al. 1998) it was demonstrated that the Ala allele of the Pro12Ala PPAR $\gamma$ polymorphism is associated with lower BMI, improves insulin sensitivity and thus reduces diabetes risk by $75 \%$. However, several subsequent reports have failed to show an association (Mancini et al. 1999; Ringel et al. 1999; Clement et al. 2000; Meirhaeghe et al. 2000), and those reports that have confirmed the association (Hara et al. 2000) have shown that the apparent protective effect of the PPAR $\gamma$ polymorphism is to a much lesser extent, which questions the role of Pro12Ala in diabetes risk. Thus, a large association study of 3000 subjects supported by a meta-analysis of sixteen previously-published studies has been conducted to overcome the inherent limitations of the smaller individual studies (Altshuler et al. 2000). This study has confirmed that the common Pro12Ala PPAR $\gamma$ polymorphism is associated with a modest $(1 \cdot 25$-fold) but significant risk of T2DM. It is interesting to note that it is the more common Pro allele (approximately $85 \%$ frequency) that is associated with T2DM and, even though the relative risk is modest (approximately1.25), the population attributable risk is high (approximately 20\%; Altshuler et al. 2000). As the Pro12Ala polymorphism introduces a missense change in the coding region of the PPAR $\gamma$ gene, it probably represents a functional variant. This hypothesis is supported by in vitro studies showing that the Ala allele decreases the DNA-binding affinity of the PPAR $\gamma$ protein and reduces its transcriptional activity (Deeb et al. 1998). Also, in human subjects loss-of-function mutations in PPAR $\gamma$ provide compelling genetic evidence that this receptor is important in the control of insulin resistance, T2DM and hypertension (Barroso et al. 1999).

Positional cloning involves mapping the susceptibility or causative loci purely on their chromosomal location using multi-generational pedigrees and/or a large number of sibling pairs. This approach allows identification of genes without any previous knowledge of biological function or the disease mechanism. Linkage and linkage disequilibrium analysis relies on the fact that genes with similar chromosome positions will only rarely be separated during genetic recombination, so susceptibility to causative genes can be localised by searching for genetic markers that cosegregate with disease. However, to date this approach has identified relatively few candidate genes relevant to the metabolic syndrome. One of the earliest important linkage peaks was shown to be at chromosome $2 \mathrm{q} 37 \cdot 3$, which has led to the identification of Calpain $10(C A P N 10)$ as a new putative diabetogene (Horikawa et al. 2000). The authors implicated an A to $\mathrm{G}$ polymorphism in intron 3 of the gene encoding CAPN1O with greater risk of T2DM. Subsequent to this initial report, several groups have shown modest associations between CAPN10 polymorphisms and haplotypes with a variety of metabolic phenotypes characteristic of the metabolic syndrome (see Florez et al. 2003). However, other groups have failed to show a relationship between CAPNIO and metabolic traits, or have shown modest associations for other sequence variations and/or haplotype combinations at the CAPN10 locus that might be associated with disease (Florez et al. 2003). The lack of a consistent gene-phenotype relationship may be related to a number of causes of inconsistency in association studies, including population-specific patterns of linkage disequilibrium, population-specific environmental triggers, genegene interactions or gene-nutrient interactions.

\section{Gene-nutrient interactions: implications for dietary fatty acid intake}

Given the complex polygenic nature of the metabolic syndrome, it is clear that understanding the nature of multiple gene-gene and gene-environment interactions is critical to understanding the pathogenesis and progression of the metabolic syndrome. In terms of addressing the gene-environment interactions, the present paper will explore the interactions between genetic background and an individual's nutrient exposure, nutritional therapy and/ or metabolic profile.

As highlighted previously, the PPAR $\gamma$ Pro12Ala polymorphism is a good candidate genetic marker relevant to the metabolic syndrome. This genetic variant also represents an excellent example of the relevance of gene-nutrient interactions in the development of the metabolic syndrome and T2DM. A recent prospective population-based cohort study of the aetiology of T2DM (Luan et al. 2001) has shown an important interaction between habitual dietary fat composition and the PPAR $\gamma$ Pro12Ala polymorphism. As the $\mathrm{P}: \mathrm{S}$ increases there is an inverse relationship for both BMI and fasting insulin concentrations in the Ala carriers but not in the Pro homozygotes. A limited interpretation of this study could be that carriers of the Pro allele, which is associated with greater risk of the metabolic syndrome, are not sensitive to dietary fat composition. Nevertheless, this study presents an important finding in relation to the potential protective effect of the Ala allele, which may be lost in the presence of a high-SFA diet. The Quebéc Family Study (Robitaille et al. 2003) has also demonstrated that the PPAR $\gamma$ Pro12Ala polymorphism modulates the relationship between dietary fat intake and components of the metabolic syndrome. In this study it was found that total fatty acid and SFA intakes are correlated with BMI, visceral adipose tissue area, waist circumference and fasting glucose concentrations in Pro12 homozygotes, but these associations are not observed among carriers of the Ala allele. Also, when the two genotype groups are classified according to quartiles of total fatty acid and SFA intakes, the positive correlation between fat and waist circumference remains in the Pro12 homozygotes, but again there is no relationship in the Ala carriers (Robitaille et al. 2003). Thus, there is a disparity between the results of these two studies that have investigated the interaction between the PPAR $\gamma$ Pro12Ala polymorphism and dietary fatty acids. However, the discrepancies may be population specific, as participants carrying the Ala allele had higher BMI than the Pro12 homozygotes in the Robitaille et al. (2003) study, an effect not observed in the study by Luan et al. (2001). Nevertheless, both studies highlight the need to take account of diet-related environmental exposure and 
the relevance of gene-nutrient interactions, particularly in relation to the possible role of dietary fatty acids in the metabolic syndrome. Also, another recent study in an Asian population (Tai et al. 2004) has failed to demonstrate this gene-nutrient interaction between the PPAR $\gamma$ Pro12Ala polymorphism and dietary fatty acid composition in a large sample cohort ( $n$ 2120). Whilst diverse genetic background and/or non-dietary environmental exposure difference between populations could account for differences, the authors suggest that lack of replication of the gene-nutrient interaction argues against the causality of this effect.

The findings of a recent randomised controlled trial (Finnish Diabetes Prevention Study; Lindi et al. 2002) suggest that the PPAR $\gamma$ Pro12Ala polymorphism could affect responsiveness to diet and exercise interventions. In this study 522 subjects with impaired glucose tolerance were randomly assigned to an intensive diet and exercise intervention group or a control group. By year 3 of the intervention the odds ratio for the development of T2DM in subjects with the Ala allele was found to be $2 \cdot 11$-fold higher compared with that for the Pro12Pro genotype. This result contrasts with other reports that show that the Ala12 allele is protective against T2DM (Altshuler et al. 2000), a discrepancy that may be partly related to differences in study design wherein earlier studies were cross-sectional and the frequency and impact of the Ala allele was compared between T2DM patients and control subjects with normal glucose tolerance. Within the dietary and exercise intervention of the Finnish Diabetes Prevention Study, which aimed to reduce body weight, Ala12 homozygotes were found to lose more weight than the Pro12Pro homozygotes, and none of the Ala12 homozygotes developed diabetes during the trial (Lindi et al. 2002).

Gene-nutrient interactions can also be modified by other non-nutrient environmental factors, such as physical activity. It has been proposed (Franks et al. 2004) that both dietary fatty acid composition and physical activity may interact with the PPAR $\gamma$ Pro12Ala polymorphism within the context of T2DM risk. Harding et al. (2004) have demonstrated that, after adjustment for BMI, physical activity level and $\mathrm{P}: \mathrm{S}$ are independently associated with lower fasting insulin concentrations and have additive effects on fasting insulin concentrations, a surrogate marker for insulin resistance. In addition, it has been shown that the interaction between $\mathrm{P}: \mathrm{S}$ and physical activity level on fasting insulin concentrations is altered when individuals are stratified according to PPAR $\gamma$ Pro12Ala genotype (Franks et al. 2004) The combined relationship between P:S and physical activity level with fasting insulin is additive in Pro12 homozygotes. In contrast, physical activity level modifies the association between P:S and fasting insulin concentrations in a multiplicative manner.

\section{Conclusions}

The recent global epidemic of the metabolic syndrome is an important illustration of how nutrient exposure and genetic background can dramatically impact on the development of the metabolic syndrome. The diverse clinical characteristics and multiple genetic targets involved in the pathogenesis and progression of the metabolic syndrome illustrate the complexity of the disease process, which involves several dysregulated metabolic pathways. Understanding the biological impact of gene-nutrient interactions is the key to understanding the basis of diet-related polygenic disorders such as the metabolic syndrome. The present paper is limited in that it focuses on the interaction between a single polymorphism in PPAR $\gamma$ and dietary fatty acid exposure, responsiveness to nutritional therapy and physical activity. It highlights the lack of consistency between studies, which may be partly ascribed to variable genetic backgrounds and/or different nutrient and non-nutrient environmental exposures. A more robust and complete understanding of the interactions between multiple genetic targets and dietary fatty acids, within the context of nutrient regulation of gene expression and individual responsiveness to dietary therapy is required. Only with a full understanding of multiple gene-gene, gene-nutrient and gene-nutrientenvironment interactions can the molecular basis of the metabolic syndrome be solved in order to reduce the risk and minimise the adverse health effects of obesity, T2DM and CVD.

\section{Acknowledgement}

Current research is supported by the EU Sixth Framework Programme Integrated Project LIPGENE (contract no. FOOD-CT-2003-505944).

\section{References}

Aguilera AA, Diaz GH, Barcelata ML, Guerrero OA \& Ros RM (2004) Effects of fish oil on hypertension, plasma lipids, and tumor necrosis factor-alpha in rats with sucrose-induced metabolic syndrome. Journal of Nutritional Biochemistry 15, 350-357.

Alberti K \& Zimmet P (1998) Definition, diagnosis and classification of diabetes mellitus and its complications. Part 1: Diagnosis and classification of diabetes mellitus, provisional report of a WHO consultation. Diabetic Medicine 15, 539-553.

Al-Shaer MH \& Abusabha H (2005) The impact of ethnicity on the lifetime risk of the metabolic syndrome and diabetes mellitus. American Journal of Cardiology 95, 819-820.

Altshuler D, Hirschhorn JN, Klannemark M, Lindgren CM, Vohl MC, Nemesh J et al. (2000) The common PPARgamma Pro12Ala polymorphism is associated with decreased risk of type 2 diabetes. Nature Genetics 26, 76-80.

Barak Y, Nelson MC, Ong ES, Jones YZ, Ruiz-Lozano P, Chien KR, Koder A \& Evans RM (1999) PPAR gamma is required for placental, cardiac, and adipose tissue development. Molecular Cell 4, 585-595.

Barroso I, Gurnell M, Crowley VE, Agostini M, Schwabe JW, Soos MA et al. (1999) Dominant negative mutations in human PPARgamma associated with severe insulin resistance, diabetes mellitus and hypertension. Nature 402, 880-883.

Barroso I, Luan J, Middelberg RP, Harding AH, Franks PW, Jakes RW, Clayton D, Schafer AJ, O'Rahilly S \& Wareham NJ (2003) Candidate gene association study in type 2 diabetes 
indicates a role for genes involved in beta-cell function as well as insulin action. Public Library of Science Biology 1, 41-55.

Brady LM, Lovegrove SS, Lesauvage SV, Gower BA, Minihane AM, Williams CM \& Lovegrove JA (2004) Increased n-6 polyunsaturated fatty acids do not attenuate the effects of longchain $n-3$ polyunsaturated fatty acids on insulin sensitivity or triacylglycerol reduction in Indian Asians. American Journal of Clinical Nutrition 79, 983-991.

Clement K, Hercberg S, Passinge B, Galan P, Varroud-Vial M, Shuldiner AR, Beamer BA, Charpentier G, Guy-Grand B, Froguel P \& Vaisse C (2000) The Pro115Gln and Pro12Ala PPAR $\gamma$ gene mutations in obesity and type 2 diabetes. International Journal of Obesity and Related Metabolic Disorders 24, 391-393.

Deeb SS, Fajas L, Nemoto M, Pihlajamaki J, Mykkanen L, Kuusisto J, Laakso M, Fujimoto W \& Auwerx J (1998) A Pro12Ala substitution in PPARgamma2 associated with decreased receptor activity, lower body mass index and improved insulin sensitivity. Nature Genetics 20, 284-287.

Expert Panel on Detection, Evaluation, and Treatment of High Blood Cholesterol in Adults (2001) Executive summary of The Third Report of the National Cholesterol Education Programme (NCEP) Expert Panel on Detection, Evaluation, and Treatment of High Blood Cholesterol in Adults (Adult Treatment Panel III). Journal of the American Medical Association 285, 2486-2497.

Fasching P, Ratheiser K, Waldhausl W, Rohac M, Osterrode W, Nowotny P \& Vierhapper H (1991) Metabolic effects of fishoil supplementation in patients with impaired glucose tolerance. Diabetes 40, 583-589.

Feskens EJ, Bowles CH \& Kromhout D (1991) Inverse association between fish intake and risk of glucose intolerance in normoglycemic elderly men and women. Diabetes Care 14, 935-941.

Feskens EJ, Virtanen SM, Rasanen L, Tuomilehto J, Stengard J, Pekkanen J, Nissinen A \& Kromhout D (1985) Dietary factors determining diabetes and impaired glucose tolerance. A 20-year follow-up of the Finnish and Dutch cohorts of the Seven Countries Study. Diabetes Care 18, 1104-1112.

Florez JC, Hirschhorn J \& Altshuler D (2003) The inherited basis of diabetes mellitus: implications for the genetic analysis of complex traits. Annual Review of Genomics and Human Genetics 4, 257-291.

Ford ES, Giles WH \& Dietz WH (2002) Prevalence of the metabolic syndrome among US adults: findings from the Third National Health and Nutrition Examination Survey. Journal of the American Medical Association 287, 356-359.

Ford ES, Giles WH \& Mokdad AH (2004) Increasing prevalence of the metabolic syndrome among U.S. adults. Diabetes 27, 2444-2449.

Franks PW, Luan J, Browne PO, Harding AH, O'Rahilly S, Chatterjee VK \& Wareham NJ (2004) Does peroxisome proliferator-activated receptor gamma genotype (Pro12ala) modify the association of physical activity and dietary fat with fasting insulin level? Metabolism 53, 11-16.

Freeman MS, Mansfield MJ, Barrett JH \& Grant PJ (2002) Heritability of features of the insulin resistance syndrome in a community-based study of healthy families. Diabetic Medicine 19, 994-999.

Groop L (2000) Genetics of the metabolic syndrome. British Journal of Nutrition 83, Suppl. 1, S39-S48.

Hara K, Okada T, Tobe K, Yasuda K, Mori Y, Kadowaki H, Hagura R, Akanuma Y, Kimura S, Ito C \& Kadowaki T (2000) The Pro12Ala polymorphism in PPAR $\gamma 2$ may confer resistance to type 2 diabetes. Biochemical and Biophysical Research Communications 271, 212-216.
Harding HA, Day NE, Khaw KT, Bingham S, Luben R, Welsh A \& Wareham NJ (2004) Dietary fat and the risk of clinical type 2 diabetes. The European Prospective Investigation of Cancer-Norfolk Study. American Journal of Epidemiology 159, 73-82.

Hirschhorn JN, Lohmueller K, Byrne E \& Hirschhorn K (2002) A comprehensive review of genetic association studies. Genetics in Medicine 4, 45-61.

Horikawa Y, Oda N, Cox NJ, Li X, Orho-Melander M, Hara M et al. (2000) Genetic variation in the gene encoding calpain-10 is associated with type 2 diabetes mellitus. Nature Genetics $\mathbf{2 6}$, $163-175$.

Isomaa B, Almgren P, Tuomi T, Forsen B, Lahti K, Nissen M, Taskinen MR \& Groop L (2001) Cardiovascular morbidity and mortality associated with the metabolic syndrome. Diabetes Care 24, 683-689.

Kahn BB \& Flier JS (2000) Obesity and insulin resistance. Journal of Clinical Investigation 106, 473-481.

Laaksonen DE, Lakkat TA, Lakkat H-M, Nyyssonent K, Rissanent T, Niskanen LK \& Salonen JY (2002) Serum fatty acid composition predicts development of impaired fasting glycaemia and diabetes in middle-aged men. Diabetic Medicine 19, 456-464.

Le Marchand-Brustel Y, Gaul P, Gremeaux T, Gonzalez T, Barres R \& Tanti JF (2003) Fatty acid-induced insulin resistance: role of insulin receptor substrate 1 serine phosphorylation in the retroregulation of insulin signalling. Biochemical Society Transactions 31, 1152-1156.

Lindi VI, Uusitupa MI, Lindstrom J, Louheranta A, Eriksson JG, Valle TT, Hamalainen H, Ilanne-Parikka P, Keinanen-Kiukaanniemi S, Laakso M \& Tuomilehto J (2002) Association of the Pro12Ala polymorphism in the PPAR-gamma2 gene with 3 -year incidence of type 2 diabetes and body weight change in the Finnish Diabetes Prevention Study. Diabetes 51, 2581-2586.

Lovejoy JC, Smith SR, Champagne CM, Most MM, Lefevre M, DeLany JP, Denkins YM, Rood JC, Veldhuis J \& Bray GA (2002) Effects of diets enriched in saturated (palmitic), monounsaturated (oleic), or trans (eladic) fatty acids in insulin sensitivity and substrate oxidation in healthy adults. Diabetes Care 25, 1283-1288.

Luan J, Browne PO, Harding AH, Halsall DJ, O'Rahilly S, Chatterjee VK \& Wareham NJ (2001) Evidence for genenutrient interaction at the PPARgamma locus. Diabetes 50, 686-689.

Mancini FP, Vaccaro O, Sabatino L, Tufano A, Rivellese AA, Riccardi G \& Colantuoni V (1999) Pro12Ala substitution in the peroxisome proliferator-activated receptor- $\gamma 2$ is not associated with type 2 diabetes. Diabetes 48, 1466-1468.

Meirhaeghe A, Fajas L, Helbecque N, Cottel D, Auwerx J, Deeb SS \& Amouyel P (2000) Impact of the peroxisome proliferator activated receptor $\gamma 2$ Pro12Ala polymorphism on adiposity, lipids and non-insulin-dependent diabetes mellitus. International Journal of Obesity and Related Metabolic Disorders 24, 195-199.

Mills GW, Avery PJ, McCarthy MI, Hattersley AT, Levy JC, Hitman GA, Sampson M \& Walker M (2004) Heritability estimates for beta cell function and features of the insulin resistance syndrome in UK families with an increased susceptibility to type 2 diabetes. Diabetologia 47, 732-738.

Perez-Jimenez F, Lopez-Miranda J, Pinillos MD, Gomez P, Paz-Rojas E, Montilla P, Marin C, Velasco MJ, BlancoMolina A, Jimenez Pereperez JA \& Ordovas JM (2001) A mediterranean and a high-carbohydrate diet improve glucose metabolism in healthy young persons. Diabetologia 44, 2038-2043. 
Popp-Snijders C, Schouten JA, Heine RJ, van der Meer J \& van der Veen EA (1987) Dietary supplementation of omega-3 polyunsaturated fatty acids improves insulin sensitivity in noninsulin-dependent diabetes. Diabetes Research 4, 141-147.

Poulsen P, Vaag A, Kyvik K \& Beck-Nielsen H (2001) Genetic versus environmental aetiology of the metabolic syndrome among male and female twins. Diabetologia 44, 537-543.

Rich SS (1990) Mapping genes in diabetes. Genetic epidemiological perspective. Diabetes 39, 1315-1319.

Ringel J, Engeli S, Distler A \& Sharma AM (1999) Pro12Ala missense mutation of the peroxisome proliferator activated receptor $\gamma$ and diabetes mellitus. Biochemical and Biophysical Research Communications 254, 450-453.

Robitaille J, Despres JP, Perusse L, \& Vohl MC (2003) The PPAR-gamma P12A polymorphism modulates the relationship between dietary fat intake and components of the metabolic syndrome: results from the Quebec Family Study. Clinical Genetics 63, 109-116.

Roche HM (2005) Fatty acids and the metabolic syndrome. Proceedings of the Nutrition Society 64, 23-29.

Roche HM \& Gibney MJ (2000) The impact of postprandial lipemia in accelerating atherothrombosis. Journal of Cardiovascular Risk 7, 317-324.
Saltiel AR (2000) The molecular and physiological basis of insulin resistance: emerging implications for metabolic and cardiovascular diseases. Journal of Clinical Investigation 106 , 163-164.

Storlien LH, Kraegen EW, Chisholm DJ, Ford GL, Bruce DG \& Pascoe WS (1987) Fish oil prevents insulin resistance induced by high-fat feeding in rats. Science $\mathbf{2 3 7}, \mathbf{8 8 5}-888$.

Tai ES, Corella D, Deurenberg-Yap M, Adiconis X, Chew SK, Tan CE \& Ordovas JM (2004) Differential effects of the C1431T and Pro12Ala PPARgamma gene variants on plasma lipids and diabetes risk in an Asian population. Journal of Lipid Research 45, 674-685.

Tanasescu M, Cho E, Manson JE \& Hu FB (2004) Dietary fat and cholesterol and the risk of cardiovascular disease among women with type 2 diabetes. American Journal of Clinical Nutrition 79, 99-105.

Trayhurn P \& Wood IS (2004) Adipokines: inflammation and the pleiotropic role of white adipose tissue. British Journal of Nutrition 92, 347-355.

Vessby B, Unsitupa M, Hermansen K, Riccardi G, Rivellese AA, Tapsell LC et al. (2001) Substituting dietary saturated for monounsaturated fat impairs insulin sensitivity in healthy men and women: The KANWU study. Diabetologia 44, 312-319. 
https://doi.org/10.1079/PNS2005445 Published online by Cambridge University Press 